\section{$\underset{\substack{\text { hommes } \\ \text { \& migrations }}}{ }$}

\section{Hommes \& migrations}

Revue française de référence sur les dynamiques

migratoires

$1313 \mid 2016$

1983, le tournant médiatique

\title{
1983, le tournant pas très cathodique
}

\section{Édouard Mills-Affif}

\section{OpenEdition \\ Journals}

\section{Édition électronique}

URL : http://journals.openedition.org/hommesmigrations/3557

DOI : 10.4000/hommesmigrations.3557

ISSN : 2262-3353

\section{Éditeur}

Musée national de l'histoire de l'immigration

\section{Édition imprimée}

Date de publication : 1 janvier 2016

Pagination : 37-43

ISBN : 978-2-919040-34-6

ISSN : $1142-852 X$

\section{Référence électronique}

Édouard Mills-Affif, « 1983, le tournant pas très cathodique », Hommes \& migrations [En ligne], 1313। 2016, mis en ligne le 01 janvier 2019, consulté le 16 mars 2020. URL : http://journals.openedition.org/ hommesmigrations/3557; DOI : https://doi.org/10.4000/hommesmigrations.3557 


\title{
1983, LE TOURNANT PAS TRĖS CATHODIQUE
}

par ÉDOUARD MILLS-AFFIF, maître de conférences en cinéma, université Paris-Diderot, et documentariste.

\author{
Les médias n'ont pas attendu l'été chaud de 1983 pour aborder \\ la complexité de la question migratoire dans leurs colonnes \\ et sur leurs antennes. Le traitement médiatique de l'immigration \\ est monté en puissance depuis les premières émeutes à \\ Vénissieux en 1981. En faisant leur apparition sur les plateaux \\ télé, les jeunes de la deuxième génération bénéficient enfin \\ d'une parole publique. Leurs mots à vif et leur sincérité \\ constituent des armes dans la lutte pour la reconnaissance \\ de leur place au sein de la société française.
}

Si 1983 est un tournant, c'est d'abord et avant tout sur le plan politique, économique et sociétal. Sur le terrain économique, le tournant (de la rigueur!) a déjà eu lieu, Jacques Delors annonçant le grand virage de la gauche dès juin 1982. Des mesures hautement symboliques, telles que le blocage des salaires et l'augmentation des taxes sur les produits de grande consommation, mettent un terme à la politique de relance. Le tournant de la rigueur signifie aussi la fermeture de sites industriels et l'annonce de plans sociaux dans les gros fleurons industriels publics (mines, métallurgie, automobile), où l'on retrouve les plus gros bataillons de la main-d'œuvre immigrée. En mars 1983, Jacques Delors, ministre de l'Économie et des Finances, présente devant un Conseil des ministres extra- ordinaire son plan de rigueur, assorti d'une batterie de mesures entraînant une ponction de 65 milliards (de francs) sur la consommation des ménages. "On reprend ce qu’on avait donné en juin 1981 », note dans ses Mémoires Jacques Attali, le conseiller du prince alors au cœur du pouvoir mitterrandien ${ }^{1}$.

C'est également en mars 1983 qu'ont lieu les élections municipales : vote sanction à l'égard du gouvernement de Pierre Mauroy, poussée de l'opposition et de l'alliance RPR-UDF et montée de l'extrême droite (à Dreux, mais aussi dans certains arrondissements parisiens et dans le Sud-Est). Ce sont, enfin, les toutes premières élections où le débat sur l'immigration (en particulier sur l'immigration clandestine) devient un enjeu central de la compétition 
politique. Face à une gauche en quête d'une culture de gouvernement et à une extrême droite renaissant de ses cendres, la droite républicaine se radicalise et fait de l'insécurité et de l'immigration ses principaux axes de campagne. Le pouvoir "socialo-communiste », comme l'appellent alors les ténors de l'opposition, est confronté à un sérieux dilemme : comment faire accepter le tournant de la rigueur auprès des militants et des électeurs

Les rodéos sont l'occasion

d'expérimenter in vivo les méthodes de l'informationspectacle, encore balbutiantes,

hésitantes, les journalistes

ne sachant pas bien où ils mettent les pieds, ni comment se positionner par rapport à ces émeutes urbaines. de gauche, sans donner l'impression d'un renoncement total à « l'esprit du 10 mai » ? La solution sera de faire le grand écart : d'un côté, le gouvernement sacrifie sans ménagement les soutiers de l'économie, les OS de Flins et de Billancourt, au nom du "réalisme économique », du "pragmatisme » et de la « modernisation de l'appareil productif », de l'autre, le Parti socialiste se fait le plus ardent défenseur des droits des immigrés, au nom des droits de l'homme et de l'antiracisme. Les questions morales sont mises en avant pour masquer le renoncement à la question sociale.

\section{Le tournant médiatique de 1981}

Sur le terrain audiovisuel, l'année 1983 marque le milieu d'une parenthèse de trois ans (19811984), au cours de laquelle les chaînes publiques (TF1, Antenne 2, FR3) ont su renouveler les traitements de l'information télévisée et ont mis à l'antenne des magazines et des documentaires de société, faisant découvrir aux téléspectateurs des univers sociaux jusque-là peu exposés médiatiquement. 1983 se situe entre deux ruptures décisives dans l'histoire de la télévision : celui de 1982 (« loi Fillioud » sur l'audiovisuel et création de la Haute Autorité) et celui de 1987 (l'année de la privatisation de TF1).
En 1983, le tournant médiatique sur l'immigration a déjà eu lieu. Dans la presse écrite, dans les Journaux télévisés, dans les reportages de société, tout ce qui, de près ou de loin, questionne la présence des immigrés dans la société française suscite de plus en plus la curiosité des médias, depuis l'arrivée de la gauche au pouvoir. Depuis les rodéos des Minguettes!

Alain Battegay et Ahmed Boubeker insistaient déjà, en 1993, dans Les Images publiques de l'immigration ${ }^{2}$, sur le fait que les émeutes de la banlieue lyonnaise en 1981 étaient l'épisode inaugural de la montée en puissance médiatique. Si la montée en Une est d'abord perceptible dans les quotidiens (Libération et Le Figaro étant les plus prolixes), les journaux télévisés de TF1 et d'Antenne 2 prennent le train en marche et se mettent à la remorque de la presse écrite. Alors que les pages "société » des grands titres de la presse couvrent les émeutes dès la mi-juillet, les journaux télévisés n'envoient des équipes sur place quà la rentrée de septembre. Les rodéos sont l'occasion d'expérimenter in vivo les méthodes de l'information-spectacle, encore balbutiantes, hésitantes, les journalistes ne sachant pas trop où ils mettent les pieds, ni comment se positionner par rapport à ces émeutes urbaines.

Un phénomène inédit en France dont chacun pensait, jusqu'ici, qu'il s'agissait d'une exclusivité "made in USA ». "Vénissieux le Bronx français? ", s'interroge Le Figaro, quelques jours après le déclenchement des premiers incidents. Les reporters de télévision se demandent à leur tour si les banlieues françaises ne seraient pas en train de prendre le chemin des ghettos noirs américains. Peur sur la ville, dans les médias de droite; crainte d'une société à deux vitesses dans les médias de gauche. Voilà comment l'on pourrait résumer schématiquement les différents regards portés sur cette actualité d'un nouveau type. Si Battegay et Boubeker considèrent que les rodéos sont le premier grand feuilleton médiatique, symptomatique des dérives et des premiers dérapages de l'information-spectacle, il convient néanmoins de nuancer. 


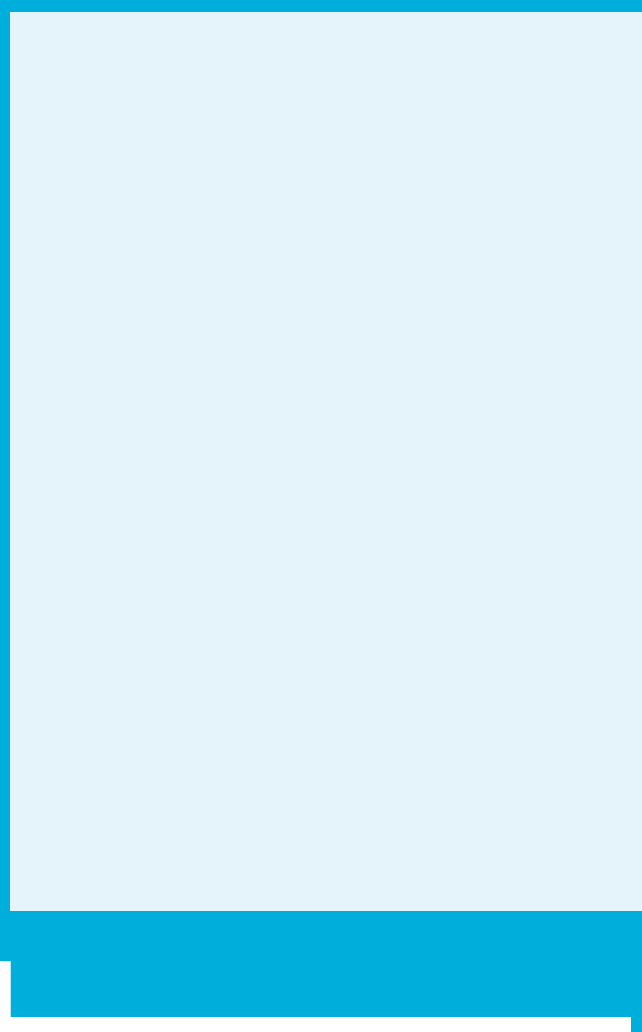

(c) AGENCE IM'MÉDIA.

Cela est vrai concernant le traitement de la presse, mais nettement moins si l'on examine attentivement la couverture des JT et les six reportages d'actualité tournés aux Minguettes dans le courant du mois de septembre 1981. Au total, si l'on additionne les reportages, les plateaux en direct et les lancements du présentateur en plateau, les JT de TF1 et d'Antenne 2 ont consacré, en tout et pour tout, une trentaine de minutes aux événements de la banlieue lyonnaise. On est donc encore très loin de l'hypermédiatisation du feuilleton sur « l'affaire des tchadors de Creil " à l'automne 1989, et de celui des émeutes de Vaulx-en-Velin, en octobre 1990. Si les rodéos des Minguettes constituent un tournant sur le plan des représentations télévisuelles de l'immigration, ce n'est pas parce que cet événement a été surexposé, ou parce qu'il aurait initié une nouvelle manière de traiter les dossiers de société, mais plutôt parce que les rodéos sont l'événement déclencheur d'un tournant iconique.

\section{Quand la banlieue s'invite au JT}

À l'été 1981, au moment des faits, alors que nous sommes encore dans la période " état de grâce " du président Mitterrand, la Haute Autorité n'est pas encore née, mais le cordon ombilical est en train d'être coupé entre la télévision et le pouvoir. Sur Antenne 2, c'est le grand retour de Pierre Desgraupes, figure historique de « l'âge d’or » de la télévi- 
sion « fenêtre sur le monde » et miroir de la société. Le premier grand chantier du nouveau patron de la Deux est la réforme des JT (Antenne 2 midi et le 20 heures). Il s'agit de donner des signaux forts du changement, de l'incarner à l'antenne, et de signifier la rupture avec la télévision giscardienne de Jean-Pierre Elkabbach et de Roger Gicquel.

Les journalistes de gauche ressortent des placards. Bernard Langlois est promu au journal de 13 heures. Le 29 septembre 1981, il présente le journal en direct de Vénissieux, en bas des tours. Sur ce plateau improvisé, en décor naturel, le maire et le préfet devaient jouer les premiers rôles, les habitants (en grande partie des jeunes, mais aussi des mères de famille) devaient être de simples figurants, à l'arrière-plan. En dépit des tentatives répétées de Langlois, appelant les habitants à laisser s'exprimer les autorités locales et à cesser leur vacarme incessant, il n'y a visiblement rien à faire pour calmer les esprits. Les figurants font irruption au devant de la scène et s'emparent du micro-baladeur, le préfet criant au scandale et quittant le pla-
Les jeunes du quartier contestent le traitement du drame dans les médias et réalisent un petit film amateur, une sorte de contreenquête, pour donner leur version des faits. teau, furibond. Pendant cinq bonnes minutes, les habitants de Vénissieux vont être les seuls maîtres à bord, sans que l'antenne soit coupée. Un tel débordement est sans précédent dans toute l'histoire de la télévision française, jusque-là obnubilée par le contrôle de la parole et la maitrise du dispositif. Après s'être enfermé dans les studios tout au long des années 1970, le petit écran retrouve le chemin du terrain. Une télévision de proximité, qui assume sa fonction de médiation sociale et qui donne la parole en priorité aux citoyens anonymes plutôt qu'aux représentants des institutions ou aux corps intermédiaires. Une télévision qui prend le risque de perdre le contrôle et de se laisser déborder. Parmi les autres journalistes de gauche d'Antenne 2, il y a Marcel Trillat, arrivé en juin 1981 pour diriger le service « société » de la rédaction. Trillat est un journaliste communiste, ex-animateur de Lorraine Coeur d'acier, radio libre financée par la CGT, depuis les grèves de la sidérurgie de 1978-1979. Deux mois après les émeutes de la banlieue lyonnaise, une bavure policière a lieu à Vitry-sur-Seine (Val-de-Marne). Kader, un jeune de 16 ans de la cité Cousy décède à l'issue d'une garde à vue. Les jeunes du quartier contestent le traitement du drame dans les médias (selon eux trop favorable à la version policière) et réalisent un petit film amateur, une sorte de contre-enquête, pour donner leur version des faits. Marcel Trillat, qui habite Vitry, récupère la vidéo et propose d'en diffuser des extraits dans le journal de 13 heures. Cela dure quatre minutes, les images sont granuleuses, les cadrages approximatifs, mais la gouaille spontanée du témoin principal (un jeune homme d'origine maghrébine à la chevelure frisée et épaisse), interviewé sur un banc devant une barre d'immeubles, est une petite tornade télévisuelle. La verve joyeusement rageuse des banlieues s'invite au journal télévisé.

Comme on a flairé les talents d'acteur du jeune homme, celui-ci est invité sur le studio du 13 heures, présenté par Patrick Lecocq. À ses côtés, sur le plateau, Guy Gilbert, «le curé des loubards », aux cheveux longs, chemise en jean et blouson noir, et Gérard Lefèvre, l'un des chroniqueurs vedettes de la rédaction d'Antenne 2. Patrick Lecocq interpelle tout d'abord Guy Gilbert : «Ces garçons qui sont avec nous aujourd'hui sur ce studio, et qui sont vraisemblablement en train de regarder à Vitry, on a du mal à les classer, hein? Politiquement, ils ne sont récupérés par personne, comment est-ce qu'on peut les classer? Ce sont des voyous, des loubards, des déracinés, plus simplement? » Guy Gilbert : "Le jeune qui est mort à Vitry était un jeune comme un autre. Ce n'était pas un loubard, ni un voyou. C'était un jeune comme un autre... » Mustapha (appelé ainsi familièrement par le présentateur) l'interrompt : "C'est les jeunes qui doivent parler, c'est nous qui devons exposer notre problème. À chaque fois qu'il y a une émission qui passe sur les jeunes, c'est toujours un psychologue machin, toujours des mecs qui connaissent rien de la jeunesse, qui 
vivent même pas dedans. "Ouais, je suis psychologue, je suis éducateur, je suis ça!” Comme lui là [il pointe du doigt Guy Gilbert]. » Mustapha est interrompu par Gérard Lefèvre : "Il y a quand même quelque chose d'important qui est la motivation des adultes qui essaient de vous comprendre. Le fait que vous soyez là en est une preuve supplémentaire. Il ne faut pas que vous adoptiez systématiquement une attitude de provocation ou de refus des adultes. Parce que c'est facile de dire "'est la faute aux autres", etc. Le problème, c'est que nous vivons tous dans la même société, que vous en acceptiez ou que vous en refusiez les règles, c'est votre problème. Nous sommes condamnés, entre guillemets, à vivre ensemble... » Mustapha : "On vit ensemble, mais toi où tu vis? On ne vit pas ensemble pour l'instant. Tu ne vis pas à Cousy, dans les ghettos de la misère... » Gérard Lefèvre: "Mais je vis dans le même pays!" Mustapha: "D'accord, dans le même pays, mais ça ne veut rien dire le même pays. Dans quelle classe tu vis, toi ? » Patrick Lecocq, sentant le ton monter, calme le jeu et reprend le contrôle de son plateau. En gros plan, face caméra : "Il y a de très nombreux téléspectateurs qui téléphonent en ce moment à la rédaction d'Antenne 2, certains pour témoigner leur accord et nous approuver de vous avoir invités aujourd'hui, d'autres, plus nombreux, je dois dire, pour exprimer leur total désaccord. Je voulais simplement dire qu'après les événements de Vitry, après la mort de Kader, et bien, il nous avait semblé juste de donner la parole à des gens qui, justement, n’ont jamais la parole à la télévision. »

\section{Une lutte pour la reconnaissance dans la société française}

Donner la parole à des gens qui ne l'ont jamais. Un défi à haut risque, Gérard Lefèvre en sort KO, et découvre, à ses dépens que, comme la sociologie, la télévision citoyenne est un sport de combat. Dernier exemple emblématique de cette télévision citoyenne, Les Dossiers de l'écran du 20 octobre 1981. Au programme ce soir-là, le film d'Yves Boisset, Dupont-Lajoie, sorti en salles en 1975 mais censuré jusque-là par le petit écran. Il s'agit donc d'une première diffusion. Après le film, comme d'habi-

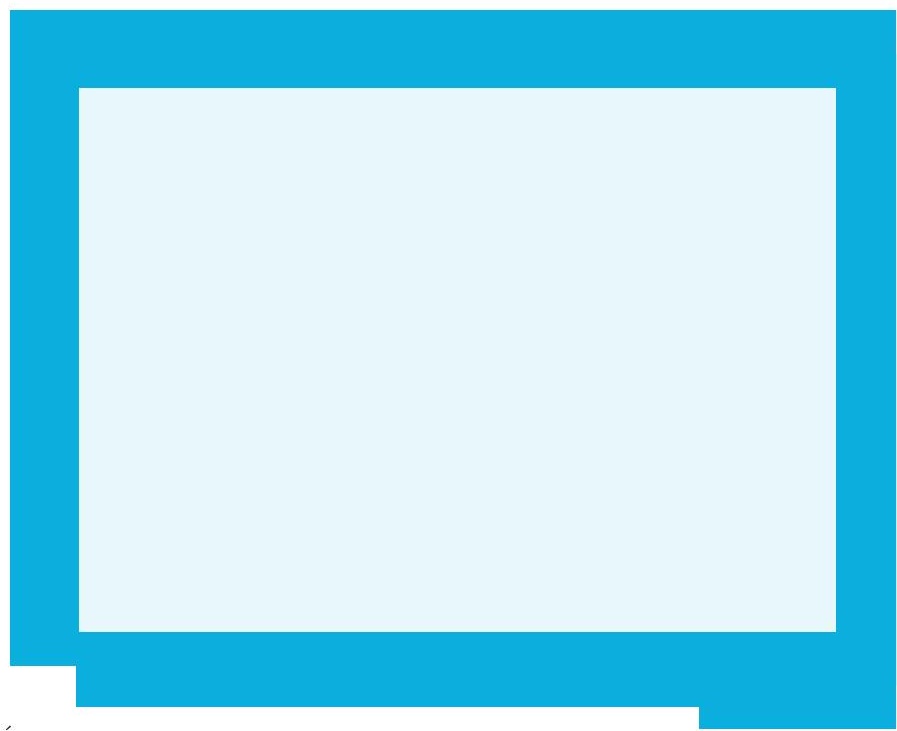

Les Minguettes, juin 1983, démolition d'une première tour, quartier Monmousseau, peu avant la visite du président François Mitterrand. (C) PHoto Benhamouda / AGENCE IM'MÉdIA.

tude, un débat contradictoire. Au milieu d'experts, d'élus locaux (dont Paul Mercieca, maire communiste de Vitry-sur-Seine, après l'affaire du foyer malien démoli au bulldozer) et de Nicole Questiaux, la ministre de la Solidarité nationale, trois jeunes de la banlieue lyonnaise, qui vont faire un coup d'éclat médiatique en prenant quasiment possession du plateau. Le débat qui devait au départ se limiter à la question du racisme s'en échappe très vite. Le premier à se lancer dans l'arène est Nourredine ${ }^{3}$. La vingtaine, il a une coupe de cheveux à la Elvis Presley et porte une chemise blanche déboutonnée jusqu'au milieu du ventre : "S'il n'y avait pas eu les rodéos, Kamel, Mohammed et moi, on aurait jamais été présents à cette émission. » L'animateur: «Et pourquoi vous faites ça? » Nourredine: 
«Ben, dites-moi, nous ça fait vingt ans quion est là en France... émigrés! En vingt ans, combien de fois on a eu l'occasion de s'exprimer? » Kamel prend le relais: «Dans tous les médias, on a vu les voitures brûler, on a dit que les jeunes de la deuxième génération provoquaient la population et tout. Mais seulement, avec les années 1980, on a découvert un nouveau problème. Le problème de tous ces jeunes de la deuxième génération. Et c'est eux qui maintenant posent un problème, soidisant. (...) Nous, les jeunes nés ici en France, on a pas demandé à naître en France, on
L'égalité des droits et des chances, la reconnaissance au droit à la France, "J'y suis j'y reste ! ", toutes les aspirations de la Marche

sont déjà exprimées ici, avec clarté et limpidité, sous

l'œil des caméras. Comme quoi, il arrive parfois au petit

écran d'être en avance sur son temps. est nés ici. Alors je ne vois pas pourquoi on pourrait nous dire de repartir. Il n'en est pas question! Et je ne demande qu'une seule chose, c'est qu'on nous respecte. Vous n'avez qu'à traverser toutes les banlieues, qu'elles soient lyonnaises, marseillaises ou parisiennes, de voir tous ces jeunes qui traînent comme ça. Même les municipalités refusent de les reconnaître... » L'animateur : "Et vous avez l'impression que c'est propre aux...aux... aux [il cherche désespérément un qualitatif approprié] citoyens d'origine maghrébine, et que cela concernerait moins d'autres ethnies qui seraient moins dans le collimateur? " Kamel : "Les premiers à être sur carte (contrôlés), ce sont les jeunes Maghrébins. En plus avec un facteur très important, la guerre d'Algérie, parce que cela ne fait que dix-neuf ans qu'elle est terminée. La guerre d'Algérie, c'est ancré chez beaucoup de gens, chez beaucoup de DupontLajoie. (...) Mon grand-père est venu ici en 14-18, il a été dans le premier contingent pour se battre ici en France. Il est rentré chez lui après avoir été amputé, estropié. Mon père a travaillé trente-six ans en France! Alors je ne vois pas au nom de quoi on va, un jour, me dire de repartir, ou m'imposer un quelconque seuil de tolérance. »

L'égalité des droits et des chances, la reconnaissance d'un droit à la France, « J'y suis j'y reste! », toutes les aspirations de la Marche sont déjà exprimées ici, avec clarté et éclat, sous l'œil des caméras. Comme quoi, il arrive parfois au petit écran d'être en avance sur son temps.

\section{Une parole spectaculaire relayée par les médias}

L'irruption soudaine de cette colère brute en train de muer en conscience politique, s'exprimant au nom d'un «nous » collectif et des valeurs de la République, préfigure l'émergence de la figure médiatique du « Beur », grande gueule rebelle à la verve à la fois rageuse et séductrice, ayant un sens aigu du show, de la représentation et de l'automise en scène. Il s'agit d'une révolution iconique : à partir des rodéos des Minguettes, la figure du jeune «Beur » de banlieue éclipse les figures traditionnelles de l'immigré, construites pendant les Trente Glorieuses et le boom migratoire des années 1960. La première génération, celle des pères, se voit, au même moment, évincée de l'économie, déclassée socialement et mise hors champ des représentations. Privées d'images susceptibles de lui redonner fierté et dignité. L'apparition des « Beurs » entraîne la disparition des immigrés.

Cela aboutit à ce paradoxe surprenant qui va s'amplifier au fil des années 1980 : plus l'immigration sera au centre des discours politiques et des joutes médiatiques, moins on verra les réalités quotidiennes des immigrés à la télévision. Si 1983 n'est pas un tournant médiatique, c'est néanmoins un point culminant de la médiatisation et, plus largement, de la visibilité croissante dans l'espace public de la deuxième génération. Entre le débat sur le «malaise des banlieues » et la délinquance urbaine, les surenchères sur l'immigration clandestine, la série de crimes racistes pendant l'été, l'onde de choc provoquée par la victoire du Front national à Dreux en septembre, la forte émotion de l'opinion suite au meurtre du Bordeaux-Vintimille en novembre, et enfin, la Marche en décembre, tous les chemins de l'actualité de 1983 semblent mener 
à la question de l'immigration et à ses répercussions dans la société française. Il n'est, par conséquent, pas surprenant que les médias se soient emparés de cette actualité foisonnante, aux allures de mille-feuille. Ce qui surprend, en revanche, c'est le nombre, beaucoup plus élevé en 1983 par rapport aux deux années précédentes, de reportages et de documentaires au long format prenant le temps de traiter plus en profondeur cette actualité multiforme. Antenne 2 a produit plus de la moitié de ces documents (13 s/ 22), suivi par FR3 (5/22) et TF1 (4/22). Cet intérêt grandissant de la Deux pour les thématiques touchant, de près ou de loin, la question de l'immigration est certes lié à la centralité croissante de ces questions dans le débat public, mais il est surtout le reflet des changements éditoriaux au sein de la chaîne.

En 1983, Pierre Desgraupes, patron d'Antenne 2 depuis près de deux ans, met en place une grille de programmes qui fait la part belle aux magazines de reportages. Après avoir réformé le JT et avoir installé à l'antenne des émissions aussi éclectiques que Cinéma cinéma, Les Enfants du rock, Aujourd'hui la vie, Aujourd'hui madame, L'Heure de vérité, Résistances, Dimanche magazine, Desgraupes entend réhabiliter le journalisme de terrain et redonner un second souffle au grand reportage. L'esprit de Cinq colonnes à la une, mais avec un nouveau ton et de nouvelles écritures. Les magazines de société sont particulièrement mis à l'honneur: Les Gens d'ici, Moi je, Remue-Méninges, Moeurs en direct, Psy show. Les cases documentaires et les magazines d'information redeviennent un enjeu de la programmation et de la compétition entre les chaînes. Cette politique des programmes porte rapidement ses fruits : avec $55 \%$ de part d'audience en 1983, la Deux coiffe pour la première fois sa rivale TF1.

TF1 tente de stopper l'hémorragie (elle a perdu 17\% d'audience en un an) en imitant les recettes de Desgraupes. Alors qu'en 1982, Les Mercredi de l'information est la seule émission d'information et de reportages sur la Une, la nouvelle grille de janvier 1983 saupoudre de magazines tous les segments de la programmation. L'après-midi, après les programmes du CNDP (essentiellement des documentaires de 26 minutes), les magazines de société Presse-citron (à 18 heures), et Le Forum du mardi (à 18 h 30). Le magazine d'information Sept sur sept, présenté par Anne Sinclair, gagne en visibilité, sa diffusion étant déplacée du samedi à 22 h 40 au dimanche à 19 heures. On note également la création de deux magazines hebdomadaires sur des faits de société : Contre-enquête (le mardi à 21 h 30), et Infovision (jeudi, 21 h 45).

Le virage est aussi sensible sur FR3, la petite dernière. À la rentrée, la chaîne crée, en prime time, deux nouvelles cases hebdomadaires réservées aux documentaires de société : Parole donnée, produite par Claude Otzenberger, et Vendredi, produit par André Campana et Jean-Charles Eleb. Enfin, la série documentaire La Vie en face, produit par Philippe Alfonsi et Patrick Pesnot, diffusée en seconde partie de soirée, clôt le dispositif. Une collection de carnets de voyages au bout de la France et d'enquêtes de terrain, réalisés à la première personne et volontairement subjectifs. Le contexte télévisuel est favorable à la multiplication d'images du réel sur cette actualité foisonnante, autour de la présence des immigrés, désormais traitée comme des faits de société, et secondairement comme des faits économiques et sociaux.

Sur le plan des représentations audiovisuelles de l'immigration, 1983 est donc l'aboutissement d'un cycle, son débouché naturel, plus qu'un point de départ. Les journalistes de télévision n'ont pas attendu la Marche pour donner la parole aux jeunes issus de l'immigration. Ils ont su flairer ce qui était en germe dans la société et qui ne demandait qu'à éclore. Le plus frappant dans l'ensemble des témoignages de la "seconde génération " recueillis à cette période, sur le terrain ou sur les plateaux en studio, reste ce désir frénétique prise de parole de la seconde génération et cette soif pressante de reconnaissance. 